b acute dystonia does not usually have subjective phenomena

c rating scales such as the Dystonia Movement Scale are very useful for detecting acute dystonia

d blepharospasm and torticollis are relatively common presentations of tardive dystonia

e the movements seen in tardive dystonia are quite distinct from those seen in idiopathic torsion dystonia.

\section{MCQ answers}

$\begin{array}{lllll}1 & 2 & 3 & 4 & 5\end{array}$

a $F$ a $T$ a $F$ a $T$ a $T$

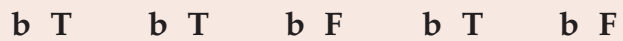

c $F$ c $F$ c $T$ c $F$ c $F$

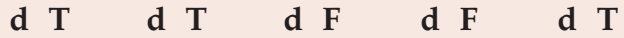

e $T$ e $T$ e $T$ e $F$ e $F$

\title{
Commentary
}

\section{G. C. Owens}

Listen for the sighs - not another overview of drugrelated motor disorders. One is often left with the uncomfortable feeling that much of mainstream psychiatry sees this as one topic they do 'know' about. Furthermore, with new-generation antipsychotics advancing apace, it relates to yesterday's problems and redundant skills.

From my own perspective as a 'teacher', it appears to me that not only are many clinicians still unclear about the nature and extent of the problem; they also often feel unprepared to give a confident and reliable evaluation of the wide range of clinical phenomena comprising these disorders, their complex manifestations and uncertain boundaries.

And what of these new drugs? Gervin and Barnes (2000) comment that they "have a lower liability for ... acute extrapyramidal side-effects (EPS)" and that while the published evidence does show this fact, controversy remains about its interpretation. Does this mean that the new antipsychotics possess an inherent and pharmacologically mediated advantage? Or, relative to comparitor drugs and regimes, do they possess an apparent and practice-mediated benefit? And regardless of the literature, does the fact that patients may prefer these new treatments 'prove' that they are indeed all they are claimed to be, or could it mean that we, as a profession, have never been as skilled as we thought in the use of the old drugs? The problems remain very much of today.

Gervin and Barnes' overview, with its emphasis on assessment, is therefore welcome - particularly in a journal devoted to 'advances'. Drug-related adverse effects would seem ideally suited to standardised monitoring as an outcome or qualityof-care measure, and evaluation and accessible recording of extrapyramidal status could become a required core skill.

It is pleasing to see the authors commenting on the subjective component of extrapyramidal dysfunction. It has always seemed ironical that while neurology has paid a deal of attention to the mental state features of parkinsonism (Cummings, 1992; Levy et al, 1998; Aarsland et al, 1999), psychiatry has been content to view drug-related disorder as almost exclusively sign led. Over the past 20 years, such narrow vision has probably been responsible

D. G. C. Owens is Professor of Clinical Psychiatry at the University of Edinburgh, Kennedy Tower, Royal Edinburgh Hospital, Morningside Terrace, Edinburgh EH10 5HF. 
for an exponentially expanding literature on 'negative' states that has produced a proportionately diminishing understanding. Gervin and Barnes point to the difficulty in quantifying disorder of this type, to which one might add the difficulty of reliable identification. None the less, it is sufficient for clinicians to be aware of the issue and to be ready to incorporate the subjective component of EPS into all relevant differentials.

The authors' statement that "rigidity of the limbs ... is perhaps the most obvious feature of druginduced parkinsonism" is not immune to challenge. Rigidity in antipsychotic-treated patients is usually mild, as the authors acknowledge with their comment on the useful role of activation. It rarely, if ever, reaches the levels of severity - and hence, certainty - found in patients with idiopathic extrapyramidal disease. It is also a uniquely difficult sign to rate confidently in those whose engagement in the examination process is tenuous. As in idiopathic Parkinson's disease, in which bradykinesia is the 'core' core disorder and necessary for the diagnosis (Quinn, 1995), bradykinesia is pervasive in drug-related disorder. The fact that it may not be obvious to psychiatrists may reflect a tendency, nurtured by training, to misattribute neurological signs to mental state disorder. One can commend bradykinesia, in all its manifestations, as 'the most obvious feature' of drug-related parkinsonism.

As Gervin and Barnes note, dystonia is not readily rateable on standard instruments. Chouinard et al's (1980) Extrapyramidal Symptom Rating Scale is one of the few scales to attempt separate ratings of dystonia, both acute and chronic. The reasons why this is laudable, if on this occasion unsuccessful, have been expounded elsewhere (Owens, 1999). This scale does, however, provide a sound means of rating parkinsonism.

The disadvantages of the professional divide between neurology and psychiatry over the past century is evident in rating scales for parkinsonism. Neurology produced over a dozen (Owens, 1999), all aimed at measuring treatment response, initially to surgery and later L-dopa. All, however, had the same problem - they were not much good (Diamond \& Markham, 1983). This led to the development of the Unified Parkinson's Disease Rating Scale (Fahn et al, 1987), now the international 'gold standard' in the field.

Psychiatry has been excluded from these developments. Unlike neurology, which acknowledged a problem and sought to address it, psychiatry remains stubbornly wedded to its own. The Neurological Rating Scale for Extrapyramidal SideEffects of Simpson and Angus (1970) was first in the field for specifically drug-related parkinsonism.
But first is not necessarily best, and the persistence and prominence of this scale in any of its numerous incarnations are both surprising and frustrating. Gervin and Barnes present some criticisms, and there are others (Owens, 1999). In my opinion, the continued use of this scale, especially at the behest of licensing authorities, does our profession no favours.

Most recording instruments for tardive dyskinesia emerged from psychiatry. Multi-item scales, such as the Tardive Dyskinesia Rating Scale (Simpson et al, 1979), can provide detailed typologies, but are complex and force recording of data in an unphysiological fashion - e.g. when evaluating 'tongue protrusion', should one also rate mouth opening, an obviously necessary procedure in protruding the tongue? However, mouth opening in this situation is totally different from the imposed contortions of primary dystonia affecting jaw musculature. Global impression scales, regionally ordered (such as the AIMS, see below), are not free from this problem, but are less afflicted by it.

All the standardised scales referred to by Gervin and Barnes were devised to serve the needs of research, and whether they automatically transfer to routine practice has never been evaluated. It is likely, however, that at least some can be usefully applied in that context as well. It is important to remember that none of these methodologies is, as is often stated, objective. All are rater subjective - a distinctly different principle. Thus, all the biases relevant to patients apply to raters too. These can probably never be eradicated completely, but they can be mitigated by two prerequisites essential to their introduction teaching and training. The first ensures that all those involved in standardised data recording are aware of, and agreed on, what precisely comprises the phenomenology to be rated. The second establishes reliabilities with the chosen instrument. Both are particularly important if multi-disciplinary rating is envisaged. Undertaking this method of data acquisition and recording without them is foolish, and it will produce a veneer of competence on a core of misleading information. Audit managers, please note!

We cannot dictate which scale(s) to choose for routine use but, as Gervin and Barnes' proposal highlights, patient evaluations must be comprehensive, covering all the syndromal features of antipsychotic-related motor dysfunction. The Abnormal Involuntary Movement Scale (AIMS; Guy, 1976) is probably rightly the 'market leader' hyperkinetic rating scale for the research community and could easily fulfil a similar function in routine practice. I find its 'one less' rule, which Gervin and Barnes appear to accept, illogical and have argued 
for its suspension (Owens, 1999). The choices for parkinsonism are less clear (especially if one excludes the Simpson-Angus scale), but a combination of AIMS and the Targeting Abnormal Kinetic Effects scale (Woijcik et al, 1980) forms a useful and undemanding package.

At a practical level, regular rating must take account of context - both 'simultaneous' (disorder in one area influencing rating in another), and 'prior' (impressions of previous individual or group disorders unduly influencing perception of present abnormality). Like most scales used in psychiatry, the majority of those for motor disorder favour a severity continuum along an uneven number of anchor points, leaving raters open to the 'central tendency' effect, that is to say, unwittingly rating down the middle. And finally, one must consider beforehand what is to be done with the information, in particular whether it is to be subjected to statistical evaluation, which itself presents a series of unresolved problems (Owens, 1999).

Standardised examination has much to commend it and is predicated on the simple principles of structure and practice. Successful standardised recording rests on teaching and training. Thus, the introduction of standardised methodologies into routine practice requires upfront investment and will have resource implications.

While standardised methods can be commended for routine use, they are not without problems of their own, and in recommending them awareness of their weaknesses is perhaps a greater asset than knowledge of their strengths.

\section{References}

Aarsland, D., Larsen, J. P., Lim, N. G., et al (1999) The range of neuropsychiatric disturbances in patients with Parkinson's Disease. Journal of Neurology, Neurosurgery and Psychiatry, 67, 492-496.

Chouinard, G., Ross-Chouinard, A., Annable, L., et al (1980) Extrapyramidal rating scale. Canadian Journal of Neurological Science, 7, 233.

Cummings, J. (1992) Depression and Parkinson's Disease. American Journal of Psychiatry, 149, 443-454.

Diamond, S. G \& Markham, C. H. (1983) Evaluating the evaluations: or how to weigh the scales of parkinsonian disability. Neurology, 33, 1098-1099.

Fahn, S., Elton, R. L. \& Members of the UPDRS Development Committee (1987) Unified Parkinson's Disease Rating Scale. In Recent Developments in Parkinson's Disease (eds S. Fahn, C. D. Marsden, M. Goldstein, et al). Florham Park, NJ: Macmillan.

Gervin, M. \& Barnes, T. R. E. (2000) Assessment of drugrelated movement disorders in schizophrenia. Advances in Psychiatric Treatment, 6, 332-341.

Guy, W. (1976) ECDEU Assessment Manual for Psychopharmacology, pp. 534-537. Washington DC: US Department of Health, Education and Welfare.

Levy, M. L., Cummings, J. L., Fairbanks, L. A., et al (1998) Apathy is not depression. Journal of Neuropsychiatry and Clinical Neuroscience, 10, 314-319.

Owens, D. G. C. (1999) A Guide to the Extrapyramidal SideEffects of Antipsychotic Drugs. Cambridge: Cambridge University Press.

Quinn, N. (1995) Parkinsonism - recognition and differential diagnosis. British Medical Journal, 310, 447-452.

Simpson, G. M. \& Angus, J. W. S. (1970) A rating scale for extrapyramidal side-effects. Acta Psychiatrica Scandinavica, Supplementum, 212, 11-19.

—, Lee, L. H., Zoubok, B., et al (1979) A rating scale for tardive dyskinesia. Psychopharmacology, 64, 171-179.

Wojcik, J. D., Gelenberg, A., La Brie, R. A., et al (1980) Prevalence of tardive dyskinesia in an outpatient population. Comprehensive Psychiatry, 21, 370-379. 